\title{
O gerente está doente. Uma perspectiva sociológica sobre os vínculos existentes entre obesidade e trabalho
}

\section{| ${ }^{1}$ Pablo Schencman |}

Resumo: Na última década, vários estudos têm-se concentrado na relação entre obesidade e emprego, abordando esta condição física como sendo de caráter incapacitante. Esses mesmos estudos conjugam as dimensões legal, administrativa e econômica no âmbito das ciências sociais, com o intuito de lançar alguma discussão sobre a temática emergente em nível histórico e que até agora não tem sido contemplada. Esta mesma matéria visa propor soluções que, num âmbito legislativo, possam proteger os obesos enquanto entidades individuais, contra a discriminação corporativa. É através deste mesmo interesse que este artigo surge, mostrando uma visão oposta e reconstruindo o vínculo que existe entre a ascensão laboral do indivíduo e, de forma paralela, o incremento de peso. A coincidência entre as trajetórias corporal e profissional surge com regularidade empírica, fruto de uma investigação mais ampla que recria as representações sociais associadas à obesidade na área metropolitana de Buenos Aires. Empregando os dados recolhidos nesse mesmo âmbito, iremos interpretá-los através da análise das mudanças nas condiçôes de trabalho que sucederam durante a última década na Argentina. Este artigo irá questionar quais os elementos que, compreendidos na atual condução dos recursos humanos, levam os trabalhadores, cuja carreira é ascendente, a uma corporalidade que, estatisticamente, é alvo de rejeição. Em segundo lugar, nos perguntamos quais são as representações associadas ao corpo que circulam entre os trabalhadores e que os unem no ingresso na obesidade juntamente com seu progresso laboral. A sensação de abandono no cuidado físico como consequência do stress e maior compromisso com a carreira profissional aparece aqui como uma das descobertas mais significativas deste artigo. Por outro lado, há coincidência entre a necessidade de valorizar o corpo como uma substância maleável, e ao mesmo tempo assumi-lo como uma consequência das opções pessoais.
1 CONICET-UBA, Buenos Aires, Argentina. Endereço eletrônico: p.schencman@gmail.com 


\section{Introdução}

O presente artigo reflete sobre uma relação dos indivíduos que contrasta com o próprio senso comum: biograficamente (os trabalhadores) compreendem e percebem sua ascensão numa escala laboral (dado mensurável, para além de sua remuneração salarial), e essa ascensão laboral é acompanhada por sua chegada à obesidade. $\mathrm{O}$ novo corpo é pensado como deteriorado em termos sociais, mas ao mesmo tempo, perfeitamente adaptado ao trabalho em que se encontra. Esta dita interseção de temas (entre o ramo da sociologia, que se dedica ao trabalho e aquele que se ocupa dos corpos) nasce a partir do trabalho de campo, orientado a reconstruir e compreender as representaçôes sociais relacionadas com o fenômeno da obesidade como um problema de saúde atual.

Historicamente, as relações entre corpo e trabalho são vastas e a evolução da produção industrial não pode ignorar a dimensão carnal no momento de apresentar suas origens como transformações posteriores. Como exemplo dessa mesma visão histórica, temos a análise de Coriat (1979), na qual ele mostra que o aparecimento da organização fabril implicou a domesticação (ou inclusivamente a criação) do corpo trabalhador. O autor coloca a oficina como o coração da organização manufatureira e afirma que a fixação espacial, a ausência de autonomia e a decisão quanto aos prazos e tempos de trabalho, bem como a atribuição de uma única tarefa, são as principais mudanças que Taylor impregna nas entidades produtivas.

As décadas que separam essa análise da atualidade geraram múltiplas alternativas na organização do trabalho, que acarretam condiçôes específicas de produção para os bens que lideram a economia atual. Por razões de pertinência, as mesmas não serão aqui discutidas, mas é necessário reconhecer que, a partir de Coriat (1979), fica cristalizada uma ligação fundamental para este estudo: aquela que associa o sofrimento físico como parte constituinte do trabalho fabril. ${ }^{1}$ A partir de um exercício de hermenêutica, podem ser reutilizadas as categorias cartesianas para distinguir entre os trabalhadores que apresentam uma condição física daqueles que colocam seu desconforto num plano psíquico. Mas, para além da geografia da dor, torna-se difícil refutar que a trajetória da produção empresarial implica uma quantidade significativa de seres que sofrem. ${ }^{2}$

De forma complementar a esta relação, encontramos que as crises econômicas, situaçôes recorrentes nas últimas três décadas na Argentina, geraram um desejo de ampliar a empregabilidade ${ }^{3}$ entre os agentes numa tentativa de evitar ser 
adicionado à percentagem de desempregados sazonais do seu país ou mesmo ser forçado a abandoná-lo. Esta dupla pressão vivida pelos trabalhadores foi abordada por Leslie \& Butz (1998), que a partir do trabalho qualitativo na planta automotriz de uma fábrica norte-americana, teorizaram sobre as formas de controle interno e externo em relação aos corpos fabris. Dessa mesma análise transcrevemos o seguinte fragmento:

It is often assumed that in a postindustrial, post-Fordist economy the primary impact of
work will no longer be on the body [...]. We argue that new production practices and
spatial configurations of work internal to the factory subject laboring bodies to different
and greater bodily risk. [...] The mechanical metaphor of a rigid, regulated, and non-
-self-adjusting unit is descriptive of a Foucauldian conception of the body in simple mo-
dernization [...]. A system of "sorting out laboring bodies" is being constructed, whereby
workers are segmented into a core group of employees possessing greater job security and
skill and a mass of peripheral employees with little job security [...]. The threat of unem-
ployment disciplines the labor force as a whole and adds a layer of psychological stress to
the laboring body. (LESLIE; BUTZ, 1998, p.361-2).

A combinação do esquema analítico tradicional, centralizado no controle interno da empresa, juntamente com as variáveis externas da mesma, ajuda a focalizar os efeitos corporais de que gozam os atuais modos de produção e gestão de recursos humanos (de agora em diante, mencionados como RH). Este esquema compreensivo se mostra eficaz para uma realidade nacional diferente, como é a Argentina.

De forma confluente, mostra-se que a obesidade em relação ao trabalho é estudada como uma condição que pode ser incapacitante. Esta leitura cria intrigas próprias, inéditas para outras configuraçōes físicas. Neste sentido, podemos ver um extrato do trabalho de Gilman:

Severe obesity is now considered a disability, though for a long time it was not. Only in 1993 did the federal Equal Employment Opportunity Commission rule that "severely obese" people could claim protection under federal statutes barring discrimination against the disabled. (...) Is obesity the end product of impairment, or is it impairment itself? If it must begin with impairment, what "organ" is "impaired"? Is it the body itself? Is it the digestive system? Is it the circulatory system? Or is it the mind, and do the obese therefore suffer from the most stigmatizing of impairments, mental illness? Is obesity a mental that is the result of an addictive personality in which food is the addiction [...]? Does the addiction indicate a lack of will? Is it physical dependency, as in heroin addiction? Is the addiction a genetically programed "error" in the human body that expresses itself in psychological desire for food or in the inability to know when one is no longer hungry? Is the impairment of obesity like a lung cancer insofar as it results from the voluntary consumption of a dangerous substance such as fat or carbohydrates? (GILMAN, 2005, p. 514-5). ${ }^{4}$ 
Essa acumulação de questões centrais para a temática, geograficamente tendenciosa pelas características epidêmicas que a obesidade tem para a América do Norte, ${ }^{5}$ ajuda a desenvolver questôes para as quais a sociologia do trabalho e do corpo colaboram com respostas próprias. No entanto, não encontramos na literatura disponível, elementos que permitam esclarecer casos em que a prosperidade laboral é acompanhada pela obesidade. ${ }^{6}$ Por esta mesma omissão, nosso trabalho é dirigido e conduzido pela metodologia interpretativa e qualitativa. ${ }^{7}$ Este artigo descreve as representações associadas ao corpo presentes entre os trabalhadores que manifestam a relação antes enunciada, e caracteriza também os modos de gestão dos corpos impostos pelo new management, a partir dos seus efeitos.

\section{Há que engolir as mágoas...}

A obesidade é uma condição de vida e, por conseguinte, as representações entre aqueles que se reconhecem sob sua égide variam de forma mais ou menos intensa, de acordo com o momento biográfico em que os agentes se encontram, bem como a matriz analítica aplicada. Um exemplo é o delineamento das observaçōes clínicas em relação ao corpo obeso. Diversas publicações médicas abordam a obesidade infantil como uma situação alarmante que complicará o panorama atual (delineado como epidêmico). ${ }^{8}$ A associação entre obesidade e corpo doente como uma constante que vai desde a infância até a velhice está repleta de dúvidas quanto aos custos do sistema de saúde, patologias e síndromes novas, mas não vai além da perspectiva de uma normalidade biométrica, que silencia a perspectiva biográfica.

Num movimento contrário foi escrito este artigo, que analisa cinco casos específicos. ${ }^{9}$ Suas trajetórias de vida e de trabalho foram reconstruídas a partir de entrevistas individuais, feitas em profundidade, realizadas pelo autor. Anteriormente, havia sido especificado que este trabalho faz parte da metodologia qualitativa num campo de estudo mediado pelo peso das estatísticas, assim, tornase necessário esclarecer que estes casos não procuram ser representativos de uma realidade maior, mas sim analisar especificamente algumas de suas peculiaridades. Como dado numérico, é importante notar que apenas dois dos casos abordados ${ }^{10}$ são desempenhados em empresas com mais de cem trabalhadores, enquanto os três restantes são empregados em organizações que não excedem 50 empregados. Esta característica nos leva a concordar com o trabalho de MacDermid et al. (1994) quando afirmam que a identificação de empresas menores, com áreas de trabalho 
menos problemáticas, é questionável. Para além do contraste entre os esquemas organizacionais onde os agentes são desenvolvidos, vemos que esta dimensão não tem qualquer efeito sobre a forma como nossos entrevistados percebem as representaçōes que seus empregadores têm sobre seus corpos.

Quanto à medição do progresso laboral, estamos cientes do debate que o conceito gera. Ignorando a controvérsia que dividiu as abordagens quantitativa e qualitativa (após a obtenção de uma medida padronizada) ${ }^{11}$, este trabalho terá em conta a trajetória laboral dos entrevistados e sua própria percepção do progresso, baseando-nos na voz de nossos entrevistados. Nesse sentido, não é relevante para este artigo se, efetivamente no ramo em que cada um deles desempenha sua atividade, a taxa de desemprego foi variando, tornando o acesso a um cargo de trabalho nas suas empresas mais difícil. O que nos interessa é que elementos quantificáveis são introduzidos como um meio para justificar a elaboração e o desenvolvimento de suas estratégias e, finalmente, para justificar suas decisões. Se o aumento de responsabilidade, sem modificação da remuneração envolvida, foi entendido como uma promoção para o entrevistado, então não refutamos sua perspectiva, mas apenas nos concentraremos nos detalhes do seu relato, nos quais o corpo dá importância às demais exigências dos outros.

Terminadas as considerações teóricas, iremos nos concentrar agora nos dados empíricos. A fim de focar os dois objetivos acima referidos, serão feitas breves apresentaçôes de cada caso antes de tomar alguns excertos textuais que permitam nossa teorização.

O primeiro destes casos é o de Aida. Sua trajetória educacional é a mais curta, se comparada aos outros entrevistados, uma vez que ela abandonou sua formação acadêmica no terceiro ano da escola secundária voluntariamente, para ir trabalhar com o pai numa loja gastronômica, que era propriedade da família. Esta decisão foi tomada contra a vontade dos pais, cuja resistência foi superada. Aida manteve seu desejo de se dedicar ao comércio e à loja da família em particular, com 16 anos de idade, e no momento em que a entrevistamos, trabalhava há 25 anos em diferentes empresas do mesmo setor. Ela descreve sua carreira laboral como ascendente, e atualmente ocupa uma posição de gestão de uma empresa desse mesmo setor, localizada na cidade de Buenos Aires. Seu peso também variou de forma crescente, desde a adolescência, quando se considerava esbelta, até ao momento da entrevista, quando se definiu como obesa mórbida, 
com possibilidade de realizar um bypass gástrico. Ela avalia sua atitude geral para com o corpo e a alimentação como "caprichosa", em que as diferentes atitudes que regem sua percepção do corpo são decididas de forma impulsiva e inconstante, acreditando que se limita a consumir simplesmente o que é "bom". ${ }^{12}$ Além disso, tentou diversos tratamentos para baixar de peso, obtendo resultados significativos na maioria, abandonando-os logo em seguida. Quanto à percepção da dimensão saúde-doença, esta se aproxima sobretudo do polo da salubridade; apesar de ter a noção de que ser obesa é equiparável a estar doente, não evoca qualquer doença grave ou cirurgia de grande porte. ${ }^{13}$ No momento da entrevista, Aida encontravase em tratamento para diminuir de peso, que tinha começado há quatro meses e cuja abordagem era estritamente nutricional. Os resultados apareciam de forma muito lenta, o que a levava a admitir que pensava em abandonar o tratamento.

Um ponto de partida para abordar o caso de Olivério é o que este considera como seu feito final: a perda recente de 70 quilos, a partir do bypass gástrico que realizou aos 44 anos de idade, oito meses antes da entrevista. Ao reconstruir o início do caminho que o levou a este ponto de viragem, ele apresenta três marcos associados ao mesmo: as múltiplas tentativas frustradas de entrar no curso de Contabilidade da Universidade de Buenos Aires, o início de sua carreira laboral na instituição bancária onde ainda hoje trabalha, e suas doses diárias de hidratos de carbono excessivas (ao contrário do estipulado pelas tabelas de Doses Diárias Recomendadas - DDR) que geraram aumento de peso constante. A incapacidade de se desenvolver no nível acadêmico não impossibilitou, em retrospectiva, sua progressão laboral. Olivério trabalhou em uma única empresa apenas e lá realizou uma carreira que começou como cadete, contando com o título acadêmico de perito mercantil, até sua posição atual como gerente de um setor dedicado à cobrança de dívidas, cujas funções incluem a gestão de um grupo de dez pessoas.

Simón, por sua vez, realiza uma leitura diferente de sua condição, enfatizando a dimensão da doença. A origem que ele encontra para a mesma está na correção de um problema glandular que acelerava seu metabolismo e o impedia de alcançar o peso ideal na adolescência. Antes de completar o ensino secundário, seu pai morreu e Simón se viu forçado a aceitar um emprego como encarregado de carga e descarga de mercadorias num armazém. Durante esse período, se interessou por culturismo físico e chegou a ficar 24 horas sem dormir para cumprir suas obrigações laborais e atléticas. Durante este período de treino intenso, conheceu 
sua esposa, com quem vive há quatro anos e tem duas filhas. Também nessa

mesma época ele retomou os estudos e obteve o seu diploma de ensino secundário. No entanto, sua filha mais nova foi afetada por uma meningite quando tinha apenas algumas semanas, o que forçou Simón a um grande esforço econômico, contraindo dívidas e aumentando suas horas de trabalho, ao mesmo tempo que desempenhava funções como vendedor de cursos superiores. Um ano após a recuperação de sua filha, sua esposa foi diagnosticada com câncer de mama e teve de ser submetida a uma operação e diversos tratamentos complementares. Todo este trajeto narrado levou Simón a ganhar 60 quilos de peso em relação a um registo prévio ao seu casamento (dados dos quais se lembra vividamente, pela sua competição atlética). Sua conceitualização sobre a obesidade tende a resumir-se na afirmação de que "las enfermedades de otros me llevaron a la mía" ${ }^{14}$ Seu tom não é de censura, mas de autossanção no que diz respeito ao nível de descuido que isso lhe causou sobre seu próprio corpo. No nível laboral, Simón dirige atualmente uma empresa que oferece recrutamento e treinamento de pessoal; fora isso, administra franquias de cursos de ensino superior e universidades em diferentes províncias argentinas. A possibilidade de alcançar estabilidade laboral, e por consequência também econômica, foi a forma que o entrevistado encontrou para assumir o conjunto de doenças de que padeceu sua família. É desta forma que entende como uma discrepância em relação a seus valores que sua ascensão laboral o tenha conduzido à obesidade. ${ }^{15}$ A partir de então, iniciou um tratamento nutricional que considera fundamentalmente um desafio intelectual. Esta predisposição surgiu de sua íntima associação entre o conhecimento aplicado, como um motor de desenvolvimento da força de trabalho, e a superação das doenças. No entanto, por não obter a diminuição de peso que esperava, foi vencido pela frustração que, em seu ponto de vista, evidencia as limitações do tratamento. Além disso, a partir da nossa análise, podemos observar que a resistência oferecida por seu próprio peso exibe a adaptação que fez da sua estrutura física às exigências laborais.

Os dois casos abordados apresentam coincidência de gênero, faixa etária e percurso educativo. ${ }^{16}$ Tanto Carla como Gabriela concluíram os estudos universitários e conseguiram emprego no ramo em que se especializaram. Dessa forma, Carla é designer de uma empresa gráfica e no momento da entrevista tinha 33 anos de idade, contando com uma década de experiência em diversas empresas do setor. Todo nosso encontro, que durou pouco mais de uma hora, foi 
acompanhado pelo led vermelho do seu BlackBerry, que reclamava sua atenção constantemente. $\mathrm{O}$ encontro foi finalmente concretizado, após várias tentativas falhadas anteriormente, a escassos metros de seu trabalho atual e com o custo de abrir mão da sua hora de almoço. Apesar de estarmos num bar que ela sugeriu algumas horas após o meio-dia, Carla optou por não comer nada, limitando-se a consumir uma Coca-Cola light pacientemente. A primeira coisa que se destaca do seu rosto são suas olheiras, duma cor escura intensa que permanecem sem nenhum vestígio de maquiagem, em oposição a suas pálpebras ou lábios. Se bem que tem dúvidas em contar a sua trajetória profissional em termos de uma carreira bem-sucedida, reconhece que atualmente já atingiu o limite das suas possibilidades. Carla trabalha desde os seus últimos anos como estudante na Universidade de Buenos Aires, e traça uma linha clara entre os primeiros estágios e os cargos que ocupou posteriormente. Suas primeiras experiências de trabalho de índole formativa são contadas como momentos de "paz e tranquilidade" quando, além disso, tinha mais tempo livre e podia dedicar um momento diário para o cuidado do seu corpo. ${ }^{17}$ No momento da entrevista, Carla rotulava-se como "gordinha", reconhecendo que já havia passado por momentos de maior peso e ansiedade em torno desta variável. Um dado significativo para este artigo está relacionado com as várias tentativas e/ou vontade de Carla de abandonar o trabalho atual e os sucessivos esforços do seu empregador para mantê-la na empresa. Nessas negociações obteve certos privilégios, como por exemplo, um grupo de assistentes, horários flexíveis, um transporte que a leva para casa quando tem de ficar até de madrugada, facilidades que lhe permitiram dar maior atenção à sua saúde.

O último caso que vamos analisar é o de Gabriela. Ela concluiu dois cursos superiores, administração e marketing, e no momento da entrevista desempenhava funções no segundo campo estudado. Gabriela inseriu-se numa empresa financeira e é responsável pela comunicação da mesma com seus clientes, assim como pela organização de eventos institucionais. Ela recria a sua chegada à obesidade como um ato voluntário. Seu relato faz a ligação entre a morte da mãe, quando ela tinha 19 anos de idade, e sua intenção de tentar isolar-se de qualquer tipo de afeto, procurando, através da sua obesidade, "não ser querida". Esta tentativa é apresentada como eficaz, vinculando o excesso de peso com seu estado civil, mas que, por sua vez, produz efeitos não considerados anteriormente e, 14 anos 
depois, decidiu recorrer a um bypass gástrico na busca de uma solução para o excesso de peso, após vários exames físicos, muitos dos quais impostos por seus empregadores, que começam a vê-la de forma insalubre. Entre este procedimento médico e o tempo de entrevista, Gabriela conseguiu perder 55 quilos e, em várias partes do seu testemunho emergiam as cirurgias plásticas que planejava para o semestre seguinte ao nosso último encontro, assim que houvesse uma diminuição maior do seu peso. Suas decisões resultam do movimento pendular entre os aspetos emocionais/afetivos, para os quais considera a obesidade como uma barreira eficaz e também da impressão das expectativas de seus empregadores. Neste sentido, tomamos um trecho de seu depoimento:

Me di cuenta una vez que fui a hacerme un control médico de la empresa, o sea, que tomé conciencia no es que no me daba cuenta sino que tomé conciencia real; y me pesaron y me dice la médica “cómo puede ser que seas tan joven y peses 120 kilos?”. Entonces yo ahi me quedé y dije "sí, es mucho", entonces me dice "¿nunca pensaste en hacer alguna dieta o algo como para bajar?", dije "no, en realidad no porque yo me siento bien". Pero me dice: "sos joven, pensá que no estás haciendo actividad fisica, que me estás contando...” porque yo le decía que de repente subia tres escalones y me moría, que no caminaba nada. "No, pero sos una chica joven no puede ser que tengas tanto sobrepeso con la edad que tenés" y eso como que me... es más como que me hizo un click cuando hizo referencia al nivel laboral, "en los lugares donde estás con público, si querés dar una imagen de empresa primero tenés que tener formada tu propia imagen". Eso me habrá herido en algún punto pero también me hizo hacer un click y decir "algo tengo que hacer".

Encontramos no depoimento de Gabriela justificativas diferentes para condições e comportamentos semelhantes. Todos os casos são apresentados como planos de carreira ascendentes que conseguem se organizar hierarquicamente, ao mesmo tempo em que entendem e sentem que cada passo a frente em sua carreira coloca seus corpos em posições mais deterioradas. Assim, a metáfora associada à destreza física, que implica escalar uma estrutura arquitetônica ou organizacional, implica, por sua vez, custo para a subsistência física. A obesidade é unanimemente considerada uma doença por nossos entrevistados, e através dela se sentem obrigados a recorrer a especialistas clínicos para ajudar a reverter a situação. Além disso, todos os casos apresentados convivem com a tensão entre as conjecturas que são atribuídas ao corpo produtivo e ao corpo saudável. Evocando novamente o trabalho de Leslie \& Butz (1998), descobrimos que esses preconceitos, ainda que circulares, não são compatíveis com a corporalidade que geram os modos empresariais atuais. Este número, longe de ser um preconceito muito difundido entre nossos entrevistados, é um elemento importante para 
recriar a origem histórica dos pressupostos sobre o corpo produtivo, tanto esbelto como racionalizado. Neste sentido, as semelhanças entre os atributos próprios do corpo fabril e das dietas podem ser encontradas no seguinte excerto da obra de Turner (1982), onde este analisa a contribuição do médico inglês George Cheyne (1671-1743) à matéria e sua influência no contexto do século XVIII:

It would be tempting to regard Cheyne's dietary management as an ascetic discipline having an "elective affinity" with the needs of nascent capitalism in subordinating the body to the routine of industrial production. The consequences of dietary management would be similar to those of Methodism. Some social historians have, for example, regarded Methodism as a religious movement which had the consequence of producing a disciplined, sober and semi-literate workforce, perfectly taylored to the production requirements of factory life. At one level, dietary management could work in the same direction of producing a sober and athletic population whose healthy bodies would not disrupt production as the result of illness following "irrational" eating and drinking habits. (TURNER, 1982, p.265).

Esse evento permite definir uma origem histórica da articulação entre a gestão da população produtiva que assumem os especialistas em RH, e a regulação dos processos vitais que sustentam os especialistas clínicos. Durante um período prolongado nos caminhos dos nossos entrevistados, a incerteza e a pressão laboral provocaram o abandono dos comportamentos recomendados pela nutrição clínica ${ }^{18}$ em prol da satisfação dos mandatos trabalhistas. Esta atitude é compensada profissionalmente, se bem que estabelece hábitos que repercutem na qualidade de vida dos trabalhadores. Por outro lado, encontramos uma constante naturalização de situações desencorajadas por nutricionistas, os que foram consultados por nossos entrevistados, tais como a ingestão de produtos panificados durante o trabalho, abolindo os espaços dedicados ao almoço ou ao jantar e a autorrecompensa através de alimentos, após a jornada laboral. Ambos os comportamentos são apresentados como necessários para combater o stress e a ansiedade, são "válvulas de escape" da tensão acumulada e resultam numa atitude socialmente aceitável de lidar com a ansiedade oral, especialmente que é causada pela recente proibição de fumar em espaços fechados. ${ }^{19} \mathrm{O}$ próprio conceito de trajetória pode tamisar alguns marcos biográficos que escondem o vínculo que tomamos como fato social consumado, tal como é a conivência do progresso laboral com a obesidade. No entanto, é conveniente retomar o depoimento de Aida, procurando momentos-chave em que é explícito que o próprio corpo é uma variável de ajuste para o modo atual de produção empresarial: 
[En un trabajo anterior] tuve una parálisis facial izquierda, por el stress que me producía

el trabajo. Quince dias antes me habian operado de vesícula y ahi bajé 10 kilos, porque cuando te operan de vesicula no podes comer nada, me sentía re-bien. Estuve una semana trabajando y se me paralizó la cara, que hasta se me caía el agua por el costado. Me deprimí tanto que engordé los diez kilos, me llevó quince días perderlos y una semana recuperarlos [...] Estaba trabajando, se me movió la parte izquierda de la cara, me quedó que no sentía nada. No podía cerrar el ojo, no podía tomar agua porque se me caía por acá [señala la comisura de sus labios], no podía hablar. Hice tratamiento, y además el nervio facial se me quedó sensible. Quedó un poco dañado, es imperceptible, pero la verdad es que fue jodido. Fue stress. En realidad, si, fue stress, y yo digo: "ah, por culpa de mi trabajo y mi jefe...”. Creo que fue un cúmulo de cosas. Porque el hecho de tener la presión de saber que te quedas sin trabajo, te comen los piojos, tiene que ver con que si en el trabajo estás mal, tenés que sacarte la bombacha igual porque si no te comen los piojos. Y esa presión a veces te enloquece un poco. Ni siquiera estamos hablando de que no te alcanza la plata. Estamos hablando de que no podes ir a trabajar porque no aguantas más la situación, y tenés que ir igual porque te comen los piojos. Vos te podes hacer muchos problemas y llegar a un nivel de stress porque no te alcanza la plata, en este caso ni siquiera es porque no te alcanza la plata, es porque cada vez que tenés que ir a trabajar te sentís mal.

Embora se deva notar que esta evocação do problema surge na entrevista como forma de relativizar o sucesso que era percebido por Aida no que diz respeito ao presente, torna-se significativo o modo como esta expressa o impacto e as repercussões físicas da pressão laboral. A associação entre humilhação e subjugação com a nudez é um elemento que permite visualizar a exposição que o regime de trabalho possui no seu sustento físico e, finalmente, como o peso é um índice irrefutável para mensurar a ansiedade produzida pelo ambiente laboral. Outra metáfora associada a este processo se encontra na necessidade de trabalhar para se poder alimentar e da possibilidade real de que a falta de emprego a converta, enquanto organismo vivo, em alimento para certos parasitas oportunistas. $\mathrm{O}$ trabalho é apresentado por ela como um protetor de situações trágicas, mas com a capacidade de penetrar, no longo prazo, o sustento de natureza física ou mesmo de gerar imobilidade do próprio.

Voltando às leituras durkheimianas que realizam Baudelot e Establet (1984), podemos afirmar que a força do fato social reside nos números das estatísticas e tira o dramatismo do detalhe, independentemente de se tratar de um caso de suicídio ou de um colapso nervoso. No entanto, entendemos que não é o mórbido ou a procura de impressionar, aquilo que nos induz a anotar relatos detalhados e minuciosos sobre estes eventos, mas sim para recuperar sua dimensão carnal em busca da compreensão dos efeitos que eles possuem na atual gestão dos recursos 
humanos ${ }^{20}$ nos agentes. É uma assunção básica para as recentes análises, que o processo de trabalho se tornou cada vez mais sedentário nas últimas cinco décadas, seguindo o ritmo da introdução da tecnologia na produção da fábrica. No entanto, tais mudanças não baniram a representação de um trabalho obreiro dinâmico e esbelto, geralmente muscular, que encarna a saúde, a responsabilidade e o valor, tanto econômica como socialmente. Esta imagem é um preconceito tingido de sépia, plantando-se nas antípodas do corpo dos trabalhadores atuais. $\mathrm{O}$ contraste surge a partir de um aumento das necessidades que a mesma impóe, e não no relaxamento dos comportamentos de controle que seriam apropriados.

Neste sentido, afirmamos que a suspensão do corpo, tanto como matéria que demanda cuidados, controle e/ou limites, é um leitmotiv empresarial desde sua criação e, na atualidade, acarreta a gênese de corpos obesos que são essenciais dentro da empresa, mas foi prejudicada subjetivamente fora dela. A vontade de reverter esta situação ocupando o tempo não laboral com atividades que permitam a perda de peso, tais como ir a um ginásio, é uma escolha que todos os nossos entrevistados fizeram, mas que em todos os casos foi posteriormente abandonada. A percepção generalizada sobre estas situações era de que, através de suas rotinas aeróbicas, procuravam desfazer aquilo que seu trabalho produzia nos seus corpos, algo demasiado contraditório para aqueles que achavam que sua carreira estava em processo de ascensão.

\section{Conclusões}

A concepção atual do management de recursos humanos gera uma visão maniqueísta, mas coincidente quanto à maneira de pensar a materialidade dos trabalhadores. Por um lado, encontra no reservatório obreiro um bem praticamente inesgotável de substituição infinita e aumenta a pressão sobre os corpos, a partir de novas técnicas que avaliam o trabalhador na sua individualidade e especificidade. Nesse contexto, o stress aparece como um mal-estar onipresente que adota um sem-número de consequências físicas concretas nos relatos recolhidos, mas cuja causa proibida reside na pressão laboral onde esta se encarna e ganha corpo.

Em outras palavras, a obesidade é uma forma de embodiment do sucesso laboral. Torna-se evidente que esta constatação não é consistente com as imagens de publicidade, ou do senso comum que circulam em torno do triunfo dentro 
da competição laboral. Este vínculo não é tido em conta quando se calcula que mais da metade da população mundial se considerada com excesso de peso, e o indivíduo é responsabilizado pelas suas doses (DDR) irrestritas, pelas suas más escolhas alimentares e sua sedentarização, ${ }^{21}$ mas não se foca no fato comum de uma maioria populacional, como é a inserção laboral.

Evidentemente, todos esses parâmetros são sociais e, portanto, historicamente variáveis, mas é sugestiva a falta de associação entre as mudanças nos métodos de produção que limitam o corpo produtivo a partir do emprego de tecnologias informáticas, e os números alarmantes que dão à obesidade o rótulo de epidemia.

\section{Referências}

AUGUST, G.P. et al. Prevention and treatment of pediatric obesity: an endocrine society clinical practice guideline based on expert opinion. The Journal of Clinical Endocrinology \& Metabolism, v.93, n.12, p. 4576-4599, Dec 1st 2008.

BAUDELOT, C.; ESTABLET, R. Durkheim et le suicide. Paris: PUF, 1984.

BOERO, N. All the news that's fat to print: the American "obesity epidemic" and the media. Qualitative Sociology, v.30, n.1, p. 41-60, 2006.

BONI, F. Framing media masculinities. European Journal of Communication, v.17, n.4, p.465-478, 2002.

CARR, D.; FRIEDMAN, M.A. Body weight and the quality of interpersonal relationships. Social Psychology Quarterly, v.69, n.2, p.127-149, 2006.

CLARK, G.; VAN DER YSBRAND, W. Work in progress? The Industrious Revolution. The Journal of Economic History, v.58, n.3, p.830-843, 1998.

COTIAT, B. L'atelier et Le chronomètre :essai sur le Taylorisme, le Fordisme et la production de masse. Paris: Christian Bourgois, 1979.

CROSNOE, R. Gender, obesity, and education. Sociology of Education, v.80, n.3, p.241260, 2007.

DEMATTIA, L.; LEE DENNEY, S. Childhood obesity prevention: successful communitybased efforts. The ANNALS of the American Academy of Political and Social Science, v.615, n.1, p.83-99, 2008.

DEVRIES, G.T. Innovations in workplace wellness: six new tools to enhance programs and maximize employee health and productivity. Compensation \& Benefits Review, v.42, n.1, p.46-51, 2009.

FISCHLER, C. Commensality, society and culture. Social Science Information, v.50, n.3-4, p.528-548, 2011. 
GILMAN, S. L. Defining disability: the case of obesity. PMLA, v.120, n.2, p.514-517, 2005. GREMILLION, H. The cultural politics of body size. Annual Review of Anthropology, v.34, p.13-32, 2005.

GROGAN, S.; RICHARDS, H. Body Image. Men and Masculinities, v.4, n.3, p.219232, 2002.

HOLT, R.I.G. Obesity: an epidemic of the twenty-first century: an update for psychiatrists. Journal of Psychopharmacology, v.19, n.6 suppl., p.6-15, 2005.

JOHNSON, M.K. Family roles and work values: processes of selection and change. Journal of Marriage and Family, v.67, n.2, p.352-369, 2005.

KIRKLAND, A. Fat rights: dilemmas of difference and personhood. New York: New York University Press, 2008.

LAVAN, H.; KATZ, M. Managing obesity: human resource managers' perspectives. Compensation \& Benefits Review, v.41, n.2, p.54-61, 2009.

LESLIE, D.; BUTZ, D. "GM suicide”: flexibility, space, and the injured body. Economic Geography, v.74, n.4, p.360-378, 1998.

LUDWIG, D.S. Childhood obesity: the shape of things to come. New England Journal of Medicine, v.357, p.2325-2327, 2007

MACDERMID, S.M. et al. Is small beautiful? work-family tension, work conditions, and organizational size. Family Relations, v.43, n.2, p.159-167, 1994.

MUNIZ TERRA, L. Carreras y trayectorias laborales: aproximaciones a su conceptualización, reconstrucción y análisis. Ponencia en ASET. In: CONGRESO DE LA ASOCIACIÓN ARGENTINA DE ESPECIALISTAS EN ESTUDIOS DEL TRABAJO, 10. Agosto 2011, CD-ROM.

NEFFA, J.C., Desempleo, pobreza y politicas sociales. Fortalezas y debilidades del Plan Jefas y Jefes de Hogar Desocupados. Buenos Aires: Miño y Dávila, 2008.

SMITH, V. New Forms of Work Organization. Annual Review of Sociology, v.23, p.315339, 1997.

TAYLOR, S.J.; BOGDAN, R. Introducción a los métodos cualitativos de investigación. Barcelona: Paidós, 1994.

TURNER, B.S. The government of the body: medical regimens and the rationalization of diet. The British Journal of Sociology, v.33, n.2, p.254-269, June 1982.

WELLS, N.M. et al. Environment, design, and obesity. Environment and Behavior, v.39, n.1, p.6-33, 2007. 
${ }^{1}$ Esse postulado se opõe ao pressuposto racionalista que, associado à Revolução Industrial, marcava um desenvolvimento civilizacional no caminho das exigências do trabalho agrário ao industrial.

${ }^{2}$ A dimensão política do mesmo processo pode ser encontrada em Gremillion (2005) e Grogan \& Richards (2002).

${ }^{3}$ A esse respeito, usamos a definição de Neffa (2008).

${ }^{4}$ A esse respeito, podem ser consultados também os trabalhos de Boni (2002) e Kirkland (2008).

${ }^{5}$ Os trabalhos de Boero (2006) e Holt (2005) são exemplos do modo em que esta etiqueta foi criada e reproduzida entre os profissionais de saúde e meios de comunicação.

${ }^{6}$ Os trabalhos de DeVries, (2009) e LaVan \& Katz (2009) se aproximam desta problemática, mas se concentram em sugerir modos de ação para os especialistas em RH das empresas.

${ }^{7}$ Neste sentido, seguimos as definições brindadas por Taylor \& Bogdan (1994: p.15-27, 100-132).

${ }^{8}$ Como exemplo, podem observar-se os seguintes trabalhos: August et al. (2008), Demattia \& Lee Denney (2008) e o de Ludwig (2007).

${ }^{9}$ Todos os nomes foram modificados com o objetivo de manter o anonimato que foi acordado com os nossos entrevistados. Os entrevistados são maiores de idade e de modo a ampliar o espectro de situações abarcadas pelo fenômeno, selecionamos dois homens e três mulheres para este trabalho.

${ }^{10}$ Aqueles que denominamos Simón e Gabriela.

${ }^{11}$ Uma análise deste debate pode ser encontrada no trabalho de Muñiz Terra (2011).

${ }^{12} \mathrm{O}$ que diz respeito às diversas interpretações culturais, no que toca aquilo que um grupo social define como alimento e a construção social do gosto pode-se consultado o trabalho de Fischler (2011).

${ }^{13}$ A única exceção que relata é a remoção da sua vesícula.

${ }^{14}$ Uma possível tradução seria: "as doenças dos outros levaram-me à minha".

${ }^{15}$ A este respeito, pode-se consultar o trabalho de Carr \& Friedman (2006).

${ }^{16}$ Estas afinidades e seu vínculo com a obesidade se encontram desenvolvidas em Crosnoe (2007).

${ }^{17}$ Nas suas próprias palavras, esse cuidado constituía-se em "ter tempo para fazer exercício, comer em horários normais, poder cozinhar as minhas refeições e se não for pedir muito, dormir mais de seis horas [risos nervosos]".

${ }^{18}$ Como exemplo das mesmas, podemos ter a dose moderada e em horários fixos, a diversidade na alimentação e especialmente a prática de exercícios aeróbicos.

${ }^{19} \mathrm{Na}$ Argentina, a dita legislação foi aprovada em 2006, e em 2011 uma nova lei eliminou os espaços fechados exclusivos para fumadores.

${ }^{20}$ Dada a vastidão do tema, tomamos os trabalhos de Clark \& Ysbrand Van Der (1998), Johnson (2005), Smith (1997) e Wells et al. (2007) como referência.

${ }^{21}$ A este respeito, podem ser observados diferentes documentos da WHO, organização pertencente à ONU, publicados na sua página web, em particular sugerimos o "esquema de feitos" que se encontra disponível em: <http://www.who.int/mediacentre/factsheets/fs311/en/index.html>. Acesso em 09 jul. 2012. 


\section{The manager is sick. A sociological perspective on the links between obesity and work}

In the last decade, several studies have focused on the relationship between obesity and employment, addressing this condition as disabling. These same studies combine legal, administrative and economic dimensions in the social sciences, so as to shed some discussion about the emerging topic in the historical level, and so far has not been contemplated. This same issue is to propose solutions that, in the legislative, may protect obese as individual entities, against corporate discrimination. It is through this common interest that this article appears, showing the opposite view and rebuilding the link between the rise of the individual labor and, in parallel, the weight increase. The coincidence between the body and professional trajectories arises with empirical regularity, the result of a wider investigation that recreates the social representations associated with obesity in the metropolitan area of Buenos Aires. Using data collected in the same context, we will interpret them through the analysis of changes in working conditions that succeeded during the last decade in Argentina. This article will question what elements, included in the current conduct of human resources, lead workers, whose career is ascending to a corporeality which, statistically, is subject to rejection. Second, we ask what are the representations associated to the body that circulate among workers and unite them in obesity along with their labor progress. The sense of abandonment in the physical care as a result of stress and greater commitment to career appears here as one of the most significant findings of this article. On the other hand, there is a coincidence between the need to enhance the body as a malleable substance, and while I took it as a result of personal choice.

Key words: obesity; labor; body; stress. 